\title{
Nuriiar Safarov
}

\section{Barbara Barbosa Neves and Frank Vetere, eds. Ageing and Digital Technology: Designing and Evaluating Emerging Technologies for Older Adults. Singapore: Springer, 2019. 335 pp. ISBN 978-981-13-3693-5.}

Nuriiar Safarov, Department of Social Policy, University of Helsinki. Address for correspondence: University of Helsinki, PO Box 54, Snellmaninkatu 14A, 00014, Helsinki, Finland.nuriiar.safarov@helsinki.fi.

Writing of this review was funded by the Strategic Research Council at the Academy of Finland (grants nos. 327145 and 327148) and the Centre of Excellence for Research on Ageing and Care (RG3 "Migration, Care and Ageing," Academy of Finland project no. 312310).

Ageing and Digital Technology: Designing and Evaluating Emerging Technologies for Older Adults, edited by Barbara Barbosa Neves and Frank Vetere, is a collection of 18 papers that describe the complexity of research and praxis of information and communication technologies co-design, implementation, evaluation, acceptance, and use by older adults. The book acknowledges the worldwide demographic trends of population ageing and technologization as well as the challenges and opportunities that they bring to societies. Digital technologies can potentially contribute to the well-being and quality of life, prolong and facilitate ageing in place, assist with physical and mental impairments, and, on the other hand, technology nonuse or lower use can be detrimental and lead to exclusion. The positive impact of technologies depends on the success and effectiveness of the design and its implementation, which are phenomena for interdisciplinary examination. This book is a unique integration of multiple fields including computer science, social sciences, engineering, and architecture, united together in an attempt to produce multisided knowledge on designing and tailoring the innovative digital devices, robots, websites, and services to the diverse needs and aspirations of older adults.

The book is well structured, as it presents several theoretical frames for research on emerging technologies and ageing (part 1), defines various methodological techniques and approaches used in studies of technology design and of older adults (part 2), outlines ethical considerations of the research in this field (part 3), and provides multiple examples of case studies (in part 4, as well as in chapters 2, 10, 11, 13 , and other chapters indirectly).

In the first chapter, Barbara Barbosa Neves and Frank Vetere provide an overview of the chapters to follow and give an overall introduction into the societal aspects of age and ageing. The complexity of the adoption and use of digital technology and the structural and agentic dimensions shaping them are briefly presented. Recognizing the bias of differentiating older adults as a separate group of people, ageing is presented as both a complex physical phenomenon and social construction.

Part 1 overall covers a wide range of theoretical conundrums existing in research on digital technologies and ageing. The concept of participatory design, or co-design, that goes through the entire book is problematized in part 1, but especially in chap- 
ter 2 where Anne Marie Kanstrup and Ann Bygholm seek to outline the intricacy of involving older adults in the technology design process. They reanalyzed initially considered unusable data collected in collaboration with frail older adults that demonstrated the importance of including end users into design development. Chapter 3 reviews the literature on the wider social problems of digital divides and inequalities, their determinants and consequences. Chapter 4, building on the previous two, brings the perspective of older adults as active agents in the adoption and domestication of new technologies. It states that gerontechnology (a recently emerged research field that investigates new technologies as regards to older persons and their needs) should not be framed merely as having to meet the existing needs and practices of older adults, but should account for the diversity of older adults, their aspirations, and creativity in the design process. Chapter 5 addresses specifically the issues of social isolation and loneliness and outlines how technologies can mitigate them at all levels of social participation. The importance of co-constructing technology is discussed in both chapters 5 and 6 even though the subject of each of these chapters is different: communication technologies and digital games, respectively.

Part 2 is a significant part of the book dealing with various methodological approaches and techniques used in digital technology design and evaluation. It focuses mostly on qualitative research approaches including data collection methods such as participant observations, focus group interviewing, experimental workshops, photo-elicitation, and many others. Two different forms of ethnographic approach are discussed in chapters 8 and 9, namely "vignethnography," where vignette and ethnography are united together, and "netnography," which stands for the ethnography conducted digitally. Chapter 7 emphasizes the participatory design as a postulate of technology development for older adults, showing how user-centered design approaches provide a better understanding of sociotechnical and contextual factors influencing adoption and use of digital technology. This chapter may also serve as a guide to the usability testing of digital technologies, applications, and websites oriented toward older adults. Next, in chapter 10 Stephanie Liddicoat and Clare Newton discuss virtual reality (VR) as a useful tool for the involvement of older adults in the architectural design of built environments.

Part 3 presents a vast variety of cases, issues, and unexpected ethical dilemmas that a researcher, practitioner, or developer might face in the design process. Chapters 11,12 , and 13 can be of great benefit for those planning research on technologies, assisting devices, and websites involving older adults (or vulnerable groups) as end users. Engaging older adults in co-design is challenging due to various ethical issues, for instance, related to the (mental) ability to provide informed consent, expression of emotions and conflicts happening on the field, or contradictions between ethical guidelines and reality of the research. Anticipating the problems and preparing detailed plans for how to act should such problems occur is vital, which is shown on the example of several case studies in chapters 11 and 13 as well as by the analysis of Canadian ethical policies and guidelines in chapter 12 .

Part 4 is a collection of case studies that shifts the focus from the mainstream studies of assistive technologies (with an exception of chapter 16, which is about 
robots assisting in rehabilitation process) to the recognition of the fact that older people can actively benefit from diverse entertainment technologies, such as those for social networking (chapters 17 and 18), VR and virtual avatars (chapter 14), and digital games (chapter 15).

Ageing and Digital Technology is immense in its scope of issues covered, including virtual reality and digital gaming, societal problems of digital divides and other inequalities, social isolation, loneliness and networking, health and disability in older age, security and privacy in digital technologies, active ageing and ageing in place, and topics associated with old-age care, such as social housing and care work. Nevertheless, the diversity of issues does not diffuse the focus of the volume. On the contrary, the book in its theoretical, methodological, empirical, and ethical discussions remains concentrated on the problems of inclusive technology design.

However, such wide-ranging scope of the book would benefit from more theorization on the age-old philosophical problem of agency versus structure. The book provides extensive empirical case studies on ageing people participating in digital society as active agents of digital technology adoption, evaluation, and design. Yet, in the third chapter, digital divides are defined as structural phenomena forcibly imposed on older adults, the view criticized for its rigidity and lack of attention to the agency and context of technology adoption (Helsper 2017). Theory of reasoned action and technology adoption theories are briefly mentioned in the same chapter, albeit not offering deep insights into the agency of older adults, which would have grounded theoretically the empirical arguments about older adults as active users of digital technology and the co-design significance.

Looking at the chapters closer, I was surprised to see chapter 6 written in a speculative and somewhat popular-science-like tone, which authors themselves recognized. This makes it stand apart from the cohesion of the book as a whole. Additionally, the volume would have benefitted from more consistency in the concepts used for people aged 65 and older. The much-criticized concept of seniors is used throughout the book; chapter 4 applies the concept of elderlies to speak about the older adults, which has been criticized previously for carrying the negative connotations of frailty and disability (Phillips, Ajrouch, and Hillcoat-Nallétamby 2010). Besides, in chapter 15 the use of quantitative analysis techniques such as Student's $t$ test (a statistical tool usually used to analyze larger data sets) on the sample of 31 people (where one person is more than 3 percent of the sample) is rather dubious.

These minor points do not undermine the enormous contribution that this book makes to the development and application of assistive and communication technologies for older people that will undoubtedly be of great interest to both scholars and designers.

\section{REFERENCES}

Helsper, Ellen Johanna. 2017. “The Social Relativity of Digital Exclusion: Applying Relative Deprivation Theory to Digital Inequalities." Communication Theory 27(3):223-242. doi:10.1111/comt.12110. Phillips, Judith, Kristine Ajrouch, and Sarah Hillcoat-Nallétamby. 2010. Key Concepts in Social Gerontology. London: SAGE. 